\title{
Generations of Internet (Past, Present and Future)
}

\author{
Eva N. Obinna \\ Dept. of Computer Science, Ignatius Ajuru \\ University of Education, Port Harcourt, Rivers \\ State, Nigeria
}

\author{
L. G. Kabari \\ Dept. of Computer Science \\ Ignatius Ajuru University of Education, Port \\ Harcourt, Rivers state, Nigeria
}

\begin{abstract}
Generations of internet began from the invention of electronic computers. Electronic computers contain resistors, transistors, capacitor, and integrated circuits. The internet is a system of interconnected computer networks that uses internet protocol suite to link devices worldwide. The internet is also network of networks that comprises of academic, public, private, government and business networks of local to global scope linked by a broad array of electronic, wireless and optical networking technologies. It connects people all over the world with minimal effort. Generations of internet past present and future is a research work that aimed at dividing internet into generations base on timeline, processing mode used, language used, technology used, system architecture used and characteristics of each of the generations of internet.
\end{abstract}

\section{General Terms}

Internet Generations

\section{Keywords}

Internet, Internet Protocol, Transmission Control Protocol(TCP), Internet Generations, Computer Networks, Electronic Computers.

\section{INTRODUCTION}

In 1969, the Department of Defence Advanced Research Projects Agency (ARPA) developed an experimental network called ARPAnet to link together four supercomputing centres for military research. This network had the many and difficult design requirements that it had to be fast, reliable, and capable of withstanding a nuclear bomb destroying any one computer centre on the network. From those original four computers, this network evolved into the sprawling network of millions of computers we know today as the internet. The internet itself is really a massive network of networks [11], [12]. There is no central Internet Incorporation to which you can connect. Essentially, it is a collection of Internet service providers (ISPs) who each operate their own networks, with their own clients, and agree to interconnect with each other and exchange packets. Many of the large ISPs sell connections to their network to smaller ISPs, some of whom again sell connections to other ISPs. Ultimately, these ISPs at all levels sell connections to individuals and corporations, who then merge their networks (or individual computers) into this larger network called the internet. While there is no exact governance of the Internet, communication standards and coordination of ISP actions are overseen by a non-profit organization called the Internet Society.

An affiliated organization, the Internet Engineering Task Force (IETF) coordinates the work of numerous committees that define Internet communication standards and research methods of explaining and improving Internet communication. The actual communication standards are referred to as RFCs (Requests for Comments) and are voluntarily adhered to by all ISPs. Internet users can be simply categorized as publishing and getting information on various subjects like marketing, management, science, new technologies, training materials, jobs, higher education, mathematics, music, games, software, etc. E-Commerce and the kind of information available in the internet can also be listed as text documents, graphic files, sound and video files, downloadable games and software, demo games and software, etc. At the speed the internet has been evolving, many predictions can be made about the internet in the future, like main communication method coming with functions of being translated automatically into the language preferred by the receiver, finding a tune through humming into the microphone, virtual tours of a house, car, etc. could become common thing. This is what the Internet has become today, starting from its modest birth in 1969, to become an indispensable service for the human race at present and will remain the same in the future.

\section{THE GROWTH OF INTERNET}

Since the early 80 's when the U.S. government began to share their network technology with the world, there has been growth on a scale that is hard to imagine and in the early 80 's there were only 213 registered hosts on the internet [1]. In 1986, this number had risen to 2,308 hosts. According to the internet society, a non-profit society that studies and promotes the use of internet there were approximately 500,000 computers or hosts, connected to the internet in 1990. At the mid of 1995, this number grew to estimated 53.85 million. More astounding is the number of people using the internet. 134 countries had full internet connection and an additional 52 countries had limited access (for example, e-mail only) in 1996[1]. Surveys performed by International Data Corporation and Matrix information and Directory Services found that as of September 1997 there were between 53 and 57 million users of the internet world wide. By January 1999 there were about 50 million internet connections worldwide and which were estimated to grow to 200 million by the year 2000. Today, the internet is a public, cooperative, and self sustaining facility accessible to hundreds of millions of people worldwide [13], [14]. Physically, the internet uses a portion of total resources of the currently existing public telecommunication networks [1]. Technically, what distinguishes the internet is its use of a set of protocols called TCP/IP (Transmission Control Protocol/ Internet Protocol). Two recent adaptations of internet technology also make use of the TCP/IP protocol [1].

The Internet Generation is divided base on Timeline, Processing Mode Used, Language Used, Technology Used, System Architecture used and their Characteristics.

\subsection{First Generation Internet}

For many internet users, electronic mail (email) has practically replaced the postal service for short written transactions. Electronic mail is the most widely used application on the Net. You can also carry on live "conversations" with other computer users, using IRC (Internet Relay chat). More recently, internet telephony 
(Intertel and Net conferencing) hardware and software allows real-time voice conservations [1]. The most widely used part of the Internet is the World Wide Web (often abbreviated "WWW" or called "the web") [15]. Its outstanding feature is a method of instant cross - referencing. In most Web Sites, certain words or phrases appear in text of a different color than the rest; often this text is also underlined when you select one of these words or phrases, you will be transferred to the site or page that is relevant to this word or phrase. Sometimes there are buttons, images, or portions of images that are "clickable", if you move the pointer over a spot on a website and the pointer changes into a hand, this indicates that you can click and be transferred to another site. Using the web, you have access to millions of pages of information. Web "surfing" is done with a web. The most popular of which are Netscape Navigator and Microsoft internet Explorer. The appearance of a particular website may vary slightly depending on the browser you use. Also, later versions of a particular browser are able to render more "bells and whistles" such as animation, virtual reality, sound, and music files, than earlier versions [1].

\subsubsection{Timeline of First Generation Internet}

First Generation Internet was conceived in the era of time sharing; in 1965.

\subsubsection{Processing Mode Used in First Generation Internet}

Processing mode used in First Generation Internet is time sharing and time slicing. Time sharing allows a central computer to be shared by a large number of users sitting at terminals. Each program is given use of the central processor for a fixed period of time. When the time is up, the program is interrupted and the next program resumes execution. This is called time slicing. A program can also be interrupted before the end of the time slice, or example it might have to wait for input. Time sharing is particularly effective when each user wants to use computer intermittently, such as for program development, which has long periods of editing followed by shortest runs. It is not suitable for the batch processing that is typical of commercial data processing or for large scale computation [5]

2.1.3 Language Used in First Generation Internet Programming language used on the micro computers that time sharing ran on is basic interpreter.

\subsubsection{Technology Used in First Generation Internet}

The device technology used in this First Generation Internet are microcomputers. Intergalactic network and the ARPANET (Advanced Research Projects Agency Network). ARPANET used packet switching to allow multiple computers to communicate on a single network.

\subsubsection{System Architecture Used in First Generation Internet}

In terms of system architecture the First Generation Internet allows large number of users.

\subsubsection{Characteristics of First Generation Internet} First Generation Internet is independent, incompatible, it has very slow backbone connectivity, its protocols were incompatible; that is vendor-developed and proprietary standards, and it is not interoperable.

\subsection{Second Generation Internet}

Second Generation Internet is a collaborative effort to develop advanced internet technology and applications vital to the research and education missions of higher education. Over 140 U.S universities working together with partners industry and government are leading the Second Generation Internet projects. Second Generation Internet is working to enable applications, such as telemedicine, digital libraries and virtual laboratories that are not possible with the technology underlying today's internet. As a project of the University Corporation for Advanced Internet Development (UCAID), the Second Generation Internet projects is not a single separate network, but rather joins member network application and engineering development efforts together with many advanced campus, regional, and national network. Universities, working with industry, government and other research and education networking organizations are addressing the major challenges facing the next generation of university networks by: first and most importantly, creating and sustaining a leading edge network capability for the national research community, directing network development efforts to enable a new generation of applications to fully exploit the capabilities of broad band networks, and working to rapidly transfer new network services and applications to all levels of educational use and to the broader internet community, both nationally and internationally [1].

\subsubsection{Timeline of Second Generation Internet}

Second Generation Internet came into play in the 1970,s after Scientists Robert Kahn and Vinton Cerf developed Transmission Control Protocol and Internet Protocol, or TCP/IP, a communications model that set standards for how data could be transmitted between multiple networks [2].

\subsubsection{Processing Mode Used in Second} Generation Internet

Processing mode used in Second Generation Internet is real time processing mode. Real time data processing is the execution of data in a short time period, providing near instantaneous output.

\subsubsection{Language Used in Second Generation Internet}

Language used in Second Generation Internet is C programming Language.

\subsubsection{Technology Used in Second Generation Internet}

Technology used in Second Generation Internet is the TCP/IP (Transmission Control Protocol/ Internet Protocol). DARPA (Defense Advanced Research Projects Agency) conducted the research that leads to the development of internet protocol suite [2]. Also technologies used in Second Generation Internet are: IPV6, multicasting, and Quality of Service (QoS) that will enable a new generation of internet application.

\subsubsection{System Architecture Used in Second Generation Internet}

The system architectural design used here is the end-to-end principle. The end-to-end principle is a design framework in computer networking. In networks design according to this principle, application-specific features reside in the communication end nodes of the network, rather than in intermediary nodes, such as gateways and routers, that exist to establish the network. 


\subsection{6 Characteristics of Second Generation} Internet

Second Generation Internet is not a separate network nor is it meant to replace the current commercial internet. It is not a physical network that will replace the internet. Its goal is to bring together institutions and resources to develop new technologies and capabilities that can then be deployed in the global internet. Second Generation Internet enables a new generation of applications. It recreate a leading edge research and education network capacity, transfer new capacities to the global production internet, demonstrates new applications that can dramatically enhance researcher's ability to collaborate and conduct experiments. The Second Generation Internet demonstrate enhanced delivery of education and other services like health care, environmental monitoring etc. by taking advantage of "virtual proximity" created by advanced applications, providing middleware and development tools. Second Generation Internet facilitate development, deployment, and operation of an affordable communications infrastructure, capable of supporting differentiated Quality of service (QoS) based on applications requirements of the research and education community [1]. It Promotes experimentation with the next generation of communications technologies, Coordinate adoption of agreed working standards and common practices among participating institutions to ensure end-to-end quality of service and interoperability. It has catalyzed partnership with governmental and private sector organizations. Second Generation Internet encourages transfer of technology from Second Generation Internet to the rest of the internet, Studies new impact of new infrastructure, services and applications on higher education and the internet community in general.

\subsection{Third Generation Internet}

The era of Third Generation Internet started in 1995, it lies with the U. S. government and academics. In this generation internet is being commercialized, privatized and has broader access capacity [4]. The Third Generation Internet created an avenue where people can be connected, share information, data transmission becomes easier. Services like online adverts, ecommerce and internet banking are easily rendered.

\subsubsection{Time Line of Third Generation Internet}

The era of Third Generation Internet started in 1995.

\subsubsection{Processing Mode Used by Third Generation Internet}

Processing mode used here is the OLTP (Online Transaction Processing) [3].

\subsubsection{Language Used in Third Generation \\ Internet}

Programming language used is JAVA and XML (Extended Markup Language). XML is a markup language that defines a set of rules for encoding documents in a format that is both human readable and machine readable.

\subsubsection{Technology Used in Third Generation Internet}

Technology used is high capacity transmission technologies, which includes 4G-LTE, 3G, Wifi and Fiber Optics.

\subsubsection{System Architecture Used in Third Generation Internet}

System architectural design used is Service - Oriented Architecture (SOA)
Standardization of Service - Oriented Technologies such as web services enables loose coupling of applications and made application integration and partner interoperability easier. An approach that is based on service orientation provides a governed library of well-architected service and information building blocks that can speed up the development of new solutions.

2.3.6 Characteristics of Third Generation Internet In Third Generation Internet, there is growth of cyber terrorism like ransom ware, DDos attacks and malware upsurge. Its internet backbone link is very fast, it has many more users, full transition ton commercial Internet and internet goes global.

\subsection{Fourth Generation Internet (The Future Generation)}

The Fourth Generation Internet seeks to provide better services, more intelligence, greater involvement and participation. It should be looking at worlds social and ethical values, interoperable and open. Internet today is key to almost any socio-economic activity [7]. It has reshaped economic and societal behaviors. 12 years from now the internet will be even more indispensable motor for socio-economic activity worldwide. It creates unlimited opportunities to access information and entertainment, to interact and enhance day to day transactions. The Fourth Generation Internet should offer more to our society and to the world in general. Its services provided should be more efficient. It is important that the future generation internet is designed for humans so it can meet its full potential for the society and economy at large [6], [8], [9]. It should be powerful, open data-driven, user-centric, interoperable platform ecosystem.

\subsubsection{Timeline of Fourth Generation Internet}

The era of Fourth Generation Internet should be from year 2020.

\subsubsection{Processing Mode Used by Fourth Generation Internet}

Processing mode used here should be a combination of real time processing mode and the OLTP (Online Transaction Processing) to form a hybrid processing mode.

\subsubsection{Language Used in Fourth Generation Internet}

Here a higher and better version of JAVA and XML (Extended Markup Language) should be used.

\subsubsection{Technology Used in Fourth Generation Internet}

Technologies like WiMax and 5G should be a stepping stone to move the society and socio-economic activity to a higher level where complete access to the internet wirelessly at speed comparable to broadband connections is given.

\subsubsection{System Architecture Used in Fourth Generation Internet}

In terms of architectural design its performance quality should be the combination of machine learning, A.I. with internet of things; which is where devices connected can be accessed and pinged by other connected devices.

\subsubsection{Characteristics of Fourth Generation Internet (Future Generation)}

Fourth Generation Internet should not be alienated but should be a right. Right to complete connection to the internet and 
complete access should be granted to every one of age. It should go beyond internet of things (IoT) where interrelated computing devices, mechanical and digital machines, objects, animals or people that are provided with unique identifiers and the ability to transfer data over a network without requiring human-to- human or human-to-computer interaction.

\section{CONCLUSION}

Conclusively internet past, present and future has brought about global change in our society and the world at large it has made communication easier and it has brought about massive improvement in the area of technology. Meetings are held and concluded without physical appearances of the individuals; it also made virtual classes possible and brought about social media. In future, this work can be extended further by incorporating how the internet of the future should provide better services, more intelligence, greater involvement, participation and how to reflect social and ethical values; free open and more interoperable. Also to incorporate how the internet of the future will respond to the needs of citizens.

\section{REFERENCES}

[1] Agarwal, A. and Jain, ER. V.K. 2013. Computers today \& tomorrow.

[2] Andrews, E. 2013. History stories, who invented the internet 2. december 18 .

[3] Kooijmans, A.L., Ramons, E., Greef, N. D., Delhumeau, D., Dillenberger, D. E., Potter, H., Williams, N. 2012 Redguide for business leads, Transaction processing: past, present, and future. Retrieved 02/02/2018 from https://books.google.com.ng>books september 28. updated 2012 2eptember 29:

[4] Robert, H. Z., Hbbes internet timeline 25, with support from zakon group LLC and OpenConf", 1993-2018:

[5] William, Y. A. 2014. The early years of academic computing (may, 2014)
[6] Next Generation Internet Initiative. 2012. Published 2012 September 23, last updated 2018 february 16: Team responsible Next-Generation Internet (unit E.3).

[7] Nwamae, B. 2017. Evaluating the performance of OLSR and DYMO in Wireless mesh Routing Protocol for Underground Mining Stress Test. International Journal Of Innovative Scientific \& Engineering Technologies Research, 5(4)(2360-896X), 30-35. Retrieved from http://seahipaj.org/journals-ci/dec2017/IJISETR/full/IJISETR-D-6-2017.pdf.

[8] Kessler B. and Viola R. 2017. Internet of Humans-How we would like the Internet of the Future to be. Digital Single Marketing. Speech (30 June 2017).

[9] Viola R. 2016. Next Generation Internet: The Internet of Humans. Digital Single Marketing (26 September, 2016).

[10] Greene, T. 1999. Internet and Intranet Computing ICEIS.

[11] Shultz, D. H. 2015. What is the difference between the World Wide Web and the Internet? (June 9 2015).

[12] Beal, V. 2018. What is Internet? Webopedia Definition.

[13] INTERNET Today, the Internet is a public, cooperative, and self-sustaining facility accessible to hundreds of millions of people worldwide. Retrieved from info of internet.blogspot.com on 22/07/2018.

[14] Towards a Public Metanetwork - Columbia University Several networks received NSF subsidy at the outset but have since become self-sufficient. ... Internet is accessible to ... Internet service providers or public retrieved from www.columbia.edu/dlc/wp/citi/citi488.html on $22 / 08 / 2018$

[15] Korper, S. and Ellis, J. 2001. The e-commerce book. San Diego: Academic. 